\title{
The fatty liver index, a simple and useful predictor of metabolic syndrome: analysis of the Korea National Health and Nutrition Examination Survey 2010-201I
}

This article was published in the following Dove Medical Press journal: Diabetes, Metabolic Syndrome and Obesity:Targets and Therapy

\author{
Ah Reum Khang \\ Hye Won Lee \\ Dongwon $\mathrm{Yi}$ \\ Yang Ho Kang \\ Seok Man Son
}

Division of Endocrinology and Metabolism, Department of Internal Medicine, Pusan National University Yangsan Hospital, Pusan National University School of Medicine, Yangsan, Republic of Korea
Correspondence: Seok Man Son Division of Endocrinology and Metabolism, Department of Internal Medicine, Pusan National University Yangsan Hospital, Pusan National University School of Medicine, 20 Geumo-ro, Yangsan, Republic of Korea

Tel +82 0553602354

Fax +82 0553602150

Email sonsm@pusan.ac.kr
Purpose: Nonalcoholic fatty liver disease (NAFLD) is the most common chronic liver disease worldwide, and it is the hepatic manifestation of metabolic syndrome (MetS). We aimed to estimate the prevalence of NAFLD defined by the fatty liver index (FLI), in order to investigate the association between FLI and metabolic disorders and to determine the cutoff value of FLI to screen for MetS.

Subjects and methods: This study utilized a national representative sample of Korean adults (the Korean National Health and Nutrition Examination Surveys) which was conducted in 2010-2011. A total of 10,107 adults aged 19 years or older were selected. NAFLD was diagnosed on the basis of an increased FLI $(\geq 60)$ after the exclusion of alcohol or viral liver disease. Results: NAFLD was identified in 1,134 subjects (age-standardized prevalence, 10.0\%). When subjects were categorized into three groups by FLI $(<20,20-59$, and $\geq 60)$, the higher FLI group showed a higher prevalence of hypertension (49.7\% vs $14.4 \%$ ), diabetes mellitus (DM; $20.4 \%$ vs $3.8 \%$ ), and MetS (74.9\% vs 7.4\%). FLI was positively associated with age, body mass index, blood pressure, hemoglobin A1c, and homeostasis model assessment of insulin resistance $(P$ for trend $<0.001)$. In the multivariate analysis, the higher FLI group had a significantly higher risk for hypertension $(\mathrm{OR}=2.92,95 \% \mathrm{CI}=2.18-3.90, P<0.001), \mathrm{DM}(\mathrm{OR}=4.38,95 \% \mathrm{CI}$ $=2.96-6.49, P<0.001)$, and MetS $(\mathrm{OR}=24.85,95 \% \mathrm{CI}=17.33-35.64, P<0.001)$. However, no increase was observed for cardiovascular disease after adjustment of other risk factors. The cutoff value of the FLI estimated to predict the presence of MetS was 20 (area under the curve 0.849 , sensitivity 0.828 , and negative predictive value $91.9 \%$ ).

Conclusion: NAFLD prevalence using FLI is significantly higher in subjects with metabolic disorder including MetS. FLI might be a useful screening tool to detect subjects who may require early management of MetS and who have a high cardiovascular risk.

Keywords: nonalcoholic fatty liver disease, metabolic syndrome, diabetes mellitus, hypertension, cardiovascular disease

\section{Introduction}

Nonalcoholic fatty liver disease (NAFLD) is the most common chronic liver disease worldwide and is the hepatic manifestation of metabolic syndrome (MetS). NAFLD is emerging as an important public health problem across the globe with an estimated prevalence of $20 \%-30 \%$ in Western communities and $90 \%$ in morbidly obese subjects. ${ }^{1,2}$ NAFLD is defined by an accumulation of liver fat $>5 \%$ per liver weight along with 
$<10 \mathrm{~g}$ of daily alcohol consumption. ${ }^{3}$ The importance of early diagnosis of NAFLD is necessary considering the fact that it may progress silently to cirrhosis, portal hypertension, and liver-related death in early adulthood. NAFLD is associated with MetS, obesity, type 2 diabetes, hyperlipidemia, ${ }^{4}$ and even with an increased risk of all-cause mortality. ${ }^{5}$ Most of the subjects with NAFLD do not present with abnormal symptoms, especially at the very early stage, which limits the early detection and prevention of NAFLD. Hence, early diagnosis of NAFLD and intervention are important; also, there is an urgent need for sensitive and specific biochemical markers for NAFLD, since the serial measurements of aspartate transaminase (AST) and alanine aminotransferase (ALT) can be misleading and cannot predict the severity or outcome accurately. ${ }^{6}$

Although the diagnosis of NAFLD requires a liver biopsy, noninvasive procedures have been developed that show adequate concordance with histological results. These include liver ultrasonography (US), computed tomography, magnetic resonance spectroscopy (MRS), and several indices such as fatty liver index (FLI). FLI is an algorithm combining body mass index (BMI), waist circumference (WC), gamma-glutamyl transferase (GGT), and triglyceride (TG) levels, and has been demonstrated to be a useful tool to predict the presence of NAFLD, since it shows high concordance with the imaging as well as histological criteria for NAFLD. ${ }^{7}$ Moreover, the FLI can also be used as a marker for the diagnosis of cardiovascular disease (CVD), since the variables of the FLI are the risk factors for CVD as well. ${ }^{8}$ This index was originally developed in a population residing in northern Italy; it also showed a high sensitivity and specificity in Taiwanese and Chinese population in predicting the presence of NAFLD. ${ }^{9-11}$

We aimed to estimate the prevalence of NAFLD defined by the FLI, and to investigate whether the FLI is associated with the MetS in Korean population. Additionally, we also evaluated the cutoff value of the FLI to screen the MetS, which is needed to start early intervention for preventing CVD.

\section{Subjects and methods Study subjects}

The Korean Ministry of Health and Welfare has been conducting the Korean National Health and Nutrition Examination Surveys (KNHANES) of the non-institutionalized Korean civilians since 1998. In brief, a stratified multistage probability sampling design was used, and the selection was based on sampling units considering the geographical area, sex, and age groups using household registries. Trained investigators conducted household surveys in the adults based on the questionnaires. Household surveys included demographic, socioeconomic, and dietary parameters as well as the medical history of each participant. This was a population-based, cross-sectional study from the KNHANES 2010-2011. All participants of the KNHANES included in the current study provided written informed consent. This nationwide survey was approved by the Institutional Review Board (IRB) of the Korea Center for Disease Control (IRB Nos 2010-02CON-21 and 2011-02CON-06-C). Among 17,476 subjects surveyed in the KNHANES 2010-2011, 13,306 subjects aged above 19 years participated in our study. Secondary causes of fatty liver were excluded, such as heavy alcohol consumption (alcohol quantity: $\geq 2$ times/week, $\geq 7$ cups for males and $\geq 5$ cups for females; $n=1,318$ ) and presence of hepatitis $B$ virus (HBV) or hepatitis $C$ virus $(n=191)$. Information on a history of hepatitis was obtained via self-administered questionnaires. A total of 10,107 subjects (3,867 males and 6,240 females) were included in the study, who presented with all the data for the FLI and the MetS.

History of CVD included a diagnosis of angina pectoris, nonfatal myocardial infarction, or nonfatal stroke, which was reported by self-administered questionnaires. Diabetes mellitus (DM) was defined as the personal history of DM, and/or taking antidiabetic medication and/or fasting plasma glucose level $>7.0 \mathrm{mmol} / \mathrm{L}$, and/or hemoglobin A1c (HbAlc) level $\geq 6.5 \%$. Hypertension was defined as taking antihypertensive medication and/or the mean blood pressure $>140 / 90 \mathrm{mmHg}$. A history of clinical events of CVD, such as acute myocardial infarction (AMI) or angina, was also evaluated as part of the health interview survey. Such events were included in the analysis if the subject had been previously diagnosed with AMI or angina by a physician.

BMI was calculated using the following formula: weight in kilograms/(height in meters ${ }^{2}$ ). Smoking status was determined by grouping the subjects as follows: non-smokers, current smokers, and ex-smokers. Non-smokers included both never-smokers and ex-smokers who had smoked under 100 cigarettes in their lifetime.

\section{Definition of MetS}

The criteria for MetS established by the National Cholesterol Education Program were used. ${ }^{12}$ MetS was defined when any three of the following were present: $\mathrm{WC}$ of $\geq 90 \mathrm{~cm}$ in males and $\geq 80 \mathrm{~cm}$ in females; TGs $\geq 150 \mathrm{mg} / \mathrm{dL}$ or history of a specific drug treatment; high-density lipoprotein (HDL)cholesterol $<40 \mathrm{mg} / \mathrm{dL}$ in males and $<50 \mathrm{mg} / \mathrm{dL}$ in females 
or history of a specific drug treatment; blood pressure $\geq 130$ $\mathrm{mmHg}$ systolic or $\geq 85 \mathrm{mmHg}$ diastolic, or history of antihypertensive treatment; and fasting plasma glucose level $\geq 100 \mathrm{mg} / \mathrm{dL}$ or history of antidiabetic treatment. Waist and hip circumferences $(\mathrm{cm})$ were measured in duplicate with an anthropometric tape while the subjects were wearing light clothing. WC was measured from the narrowest point between the lower border of the rib cage and the iliac crest with the subject standing and breathing normally. Blood pressure was measured using an automated technique while the subjects were in a sitting position after 20 minutes of rest.

\section{The FLI}

The FLI was estimated using the algorithm which was reported by Bedogni et al in $2006 .{ }^{9}$ This was developed in an Italian population aged $18-75$ years (61\% males), including 216 individuals with and 280 individuals without suspected liver disease.

$\mathrm{FLI}=\left(\mathrm{e}^{(0.953 \times \ln (\mathrm{TG})+0.139 \times \mathrm{BMI}+0.718 \times \ln (\mathrm{GGT})+0.053 \times \mathrm{WC}-15.745)}\right) /\left(1+\mathrm{e}^{(0.953 \times 1}\right.$ $\mathrm{n}(\mathrm{TG})+0.139 \times \mathrm{BMI}+0.718 \times \ln (\mathrm{GGT})+0.053 \times \mathrm{WC}-15.745)) \times 100$

where TG denotes TGs (mg/dL), GGT is $\gamma$-glutamyl transferase $(\mathrm{U} / \mathrm{L})$, and $\mathrm{WC}$ is the waist circumference $(\mathrm{cm})$.

The FLI ranges from 0 to 100 ; Bedogni et al ruled out fatty liver disease by an FLI $<30$ and confirmed the presence of disease with an FLI $\geq 60$ in the study population with a good diagnostic accuracy (area under the receiver operating characteristic $[$ ROC] curve $[$ AUC] $=0.85 ; 95 \% \mathrm{CI}=0.81-0.88$ ). We revised the original cutoff scores (with FLI $<20$, likelihood of $>91 \%$ for the absence of fatty liver disease; and with FLI $\geq 60$, likelihood for $>78 \%$ for the presence fatty liver disease) with reference to a previous study. ${ }^{8}$ Liver US was not included in the baseline examination, and the NAFLD diagnosis was primarily based on FLI only in the current study.

\section{Biochemical analysis}

Cholesterol, fasting glucose, creatinine, AST, and ALT were estimated using a Hitachi automatic analyzer 7600 (Hitachi Corp., Tokyo, Japan). The HbAlc was estimated with an HLC-723G7 analyzer (Tosoh Corp., Tokyo, Japan). Fasting insulin levels were estimated using an immunoradiometric assay (IRMA) technology (INS-IRMA; Bio-Source, Nivelles, Belgium). The homeostasis model assessment of insulin resistance (HOMA-IR) was calculated using the following equation: [fasting plasma insulin $(\mathrm{mIU} / \mathrm{L}) \times$ fasting plasma glucose $(\mathrm{mg} / \mathrm{dL}) \times 405]$. The HBV antigens were determined by using an E-170 assay (Roche, Mannheim, Germany).

\section{Statistical analysis}

Data are represented as the estimated means (95\% CI), unless otherwise indicated. The skewed variables were logarithmically transformed to improve the normality before the analysis.

We explored the differences between the risk factors for DM, hypertension, and CVD depending on the tertiles of FLI and the presence or absence of MetS. Complex sample analysis was used for weighting all the values of the KNHANES data following the statistical guidance from the Korea Centers for Disease Control and Prevention. Multivariate linear regression models were used to investigate the relationship between the dependent and independent variables, including potential influential factors. Association between the FLI and MetS or DM or hypertension or CVD was analyzed using the logistic regression analysis. The data are expressed as the OR with 95\% CI. The predictive accuracy of the FLI for the MetS was evaluated using the ROC curve analysis by DeLong method. The AUC with $95 \%$ CI was calculated for different markers. The ROC estimated the cutoff score of the FLI to predict the MetS. All the analyses were carried out using SPSS, version 18.0. A $P<0.05$ was considered statistically significant.

\section{Results}

A total of 10,107 subjects (mean age, 46.0 years; and male, $40 \%$ ) were analyzed. Using the FLI ( $\geq 60)$, NAFLD was identified in 1,134 (age-standardized prevalence, 10.0\%) of the 10,107 subjects, and the prevalence of NAFLD was much higher in males than that in females $(18.4 \%$ vs $6.8 \%, P<0.001)$.

The prevalence of NAFLD was much higher expectedly in the diabetic subjects as compared to the non-diabetic subjects $(29.2 \%$ in patients with DM vs $10.3 \%$ in those without DM). The mean FLI was much higher in the patients with DM $(42.3 ; 95 \% \mathrm{CI}=40.0-44.5)$ as compared to that in the patients without DM (23.6; 95\% CI =22.9-24.3). The prevalence of NAFLD was also higher in the subjects with hypertension than that in the subjects without hypertension (22.6\% in the patients with hypertension vs $8.1 \%$ in those without hypertension). The mean FLI was much higher in the patients with hypertension $(37.9$; 95\% CI $=36.6-39.2)$, which decreased during the hypertensive stage; the mean FLI in the patients with prehypertension was 29.4 (95\% CI $=28.0-30.7)$ and in the patients without hypertension was $16.1(95 \% \mathrm{CI}=1.4-16.9)$.

The prevalence of NAFLD increased with the BMI ( $2.2 \%$ in non-obese subjects and $34.0 \%$ in obese subjects). The prevalence of NAFLD showed an increasing trend with the age $(6.0 \%$ in the age of $20 \mathrm{~s}, 13.1 \%$ in the age of $60 \mathrm{~s})$. A little different prevalence pattern was observed between 
the male and female subjects (Figure 1). The prevalence of NAFLD increased with age in the females, while the highest prevalence rate in males was observed in their middle ages, especially in the $40 \mathrm{~s}$.
Table 1 presents the baseline characteristics of the subjects according to the presence of MetS. The prevalence of NAFLD was extremely higher in the subjects with MetS than that in those without MetS $(33.0 \%$ in the patients with MetS

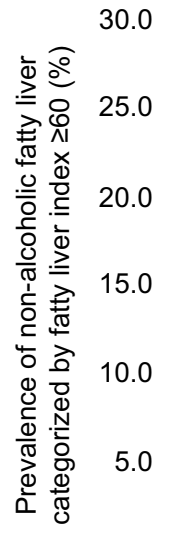

0.0

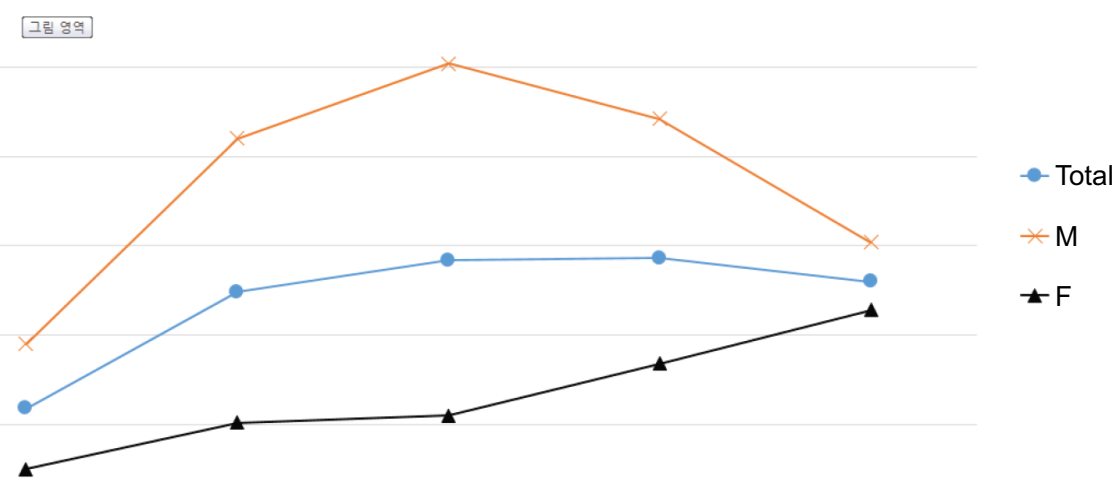

$\begin{array}{llll}19-29 & 30-39 & 40-49 & 50-59\end{array}$

Age, years

Figure I Sex difference of age-related prevalence of NAFLD.

Abbreviation: NAFLD, nonalcoholic fatty liver disease.

Table I Baseline clinical and biochemical characteristics of the study subjects according to the presence of metabolic syndrome

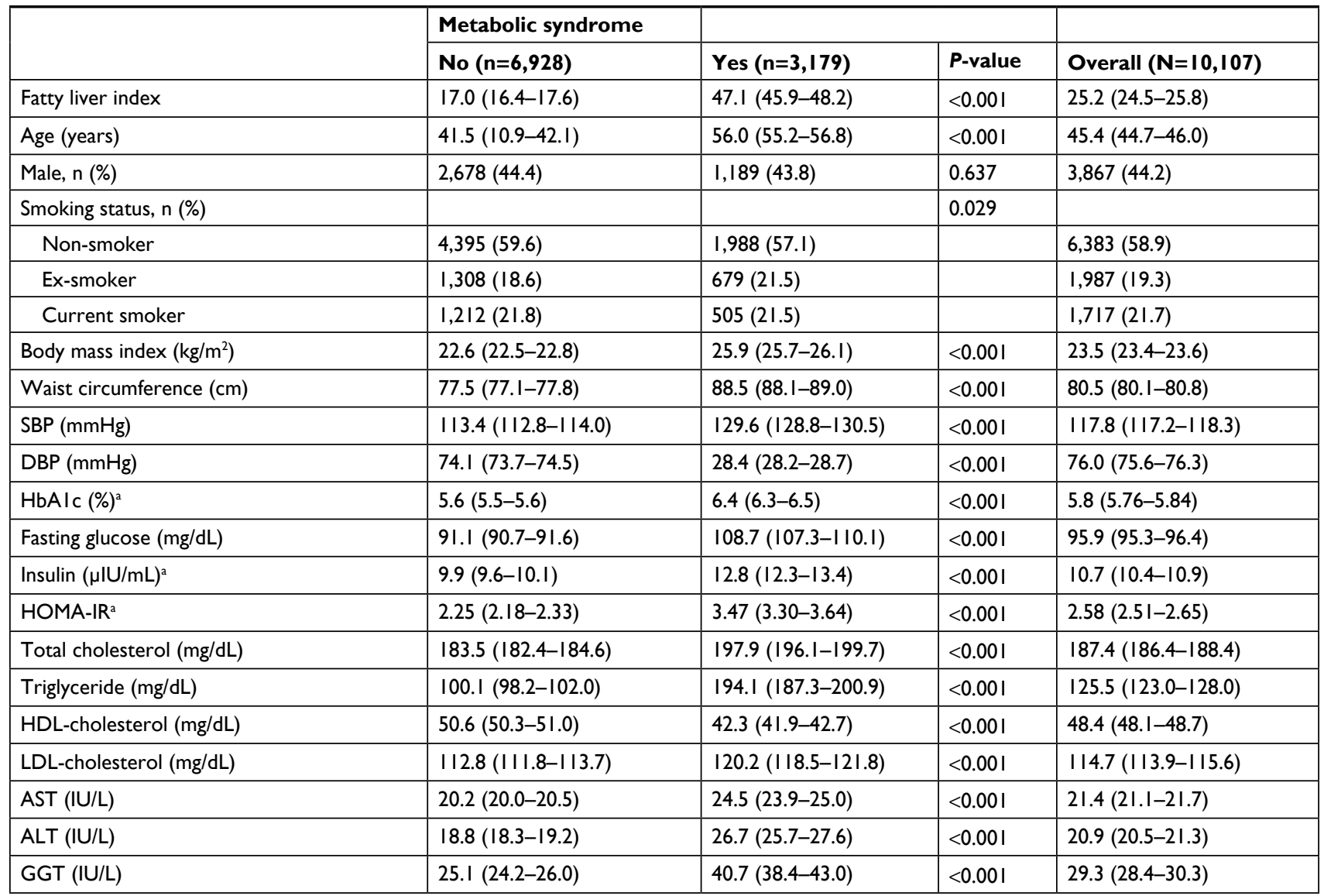

Note: ${ }^{\mathrm{Hb}} \mathrm{bIc}$, insulin, and HOMA-IR were tested only in patients with diabetes in the Korean National Health and Nutrition Examination Surveys 2010 and in all adults in the Korean National Health and Nutrition Examination Surveys 201 I.

Abbreviations: GGT, gamma-glutamyl transferase; HbAlc, hemoglobin Alc; HDL, high-density lipoprotein; HOMA-IR, homeostasis model assessment of insulin resistance; LDL, low-density lipoprotein. 
vs $4.1 \%$ in those without MetS). The mean FLI was much higher in the patients with MetS $(47.1 ; 95 \% \mathrm{CI}=45.9-48.2)$ as compared to that in the patients without MetS (17.0;95\% CI $=16.4-17.6)$. The patients with MetS presented higher low-density lipoprotein-cholesterol (LDL-C) as compared to those without MetS (120.2 vs $112.8 \mathrm{mg} / \mathrm{dL}, P<0.001$; Table 1). BMI, fasting glucose, and blood pressure were significantly higher in the patients with MetS $(P<0.001$; Table 1). The patients with MetS showed high AST, ALT, GGT, HbA1c, and HOMA-IR $(P<0.001)$.

Table 2 presents the baseline clinical and biochemical characteristics categorized by the FLI. The subjects in the third category of the FLI $(\geq 60)$ presented lower HDL-cholesterol and higher LDL-C as compared to those in the first category of the FLI (HDL-C/LDL-C: 41.7/121.4 $\mathrm{mg} / \mathrm{dL}$ in the FLI $\geq 60$ group vs $51.9 / 109.0 \mathrm{mg} / \mathrm{dL}$ in the FLI $<30$ group, $P$ for trend $<0.001$; Table 2). BMI, WC, blood pressure, and lipid profiles, except HDL-cholesterol, were positively associated with the FLI ( $P$ for trend $<0.001$; Table 2). AST, ALT, GGT, fasting glucose, HbAlc, and HOMA-IR significantly increased with increased FLI $(P$ for trend $<0.001$ ).

Table 3 shows the prevalence rates of hypertension, DM, MetS, and CVD in each FLI category. The prevalence of these metabolic diseases has already become much higher for the FLI score ranging from 20 to 60 as compared to that for the FLI of $<20$. Increasing FLI by 10 increased the risk of DM by $31 \%$, hypertension by $20 \%$, and MetS by $105 \%$ after adjusting the age, sex, BMI. Although the prevalence of CVD was also reported to be higher in the subjects with FLI $\geq 20$, there was no evidence of significantly increased risk of CVD with increasing FLI by 10 .

The OR for hypertension, DM, MetS, and CVD according to the categories of the FLI are shown in Table 4. In model 1 , the subjects in the third category $(\geq 60)$ presented an OR of $5.57(95 \% \mathrm{CI}=4.56-6.80)$ for hypertension, 5.49

Table 2 Baseline clinical and biochemical characteristics of the study subjects categorized by FLI

\begin{tabular}{|c|c|c|c|c|c|c|c|}
\hline & $\begin{array}{l}\text { FLI <20 } \\
(n=5,565)\end{array}$ & $95 \% \mathrm{Cl}$ & $\begin{array}{l}\text { FLI } 20-59 \\
(n=3,408)\end{array}$ & $95 \% \mathrm{Cl}$ & $\begin{array}{l}\text { FLI } \geq 60 \\
(n=I, 134)\end{array}$ & $95 \% \mathrm{Cl}$ & $\begin{array}{l}P \text { for } \\
\text { trend }\end{array}$ \\
\hline FLI & 7.88 & $7.70-8.06$ & 36.88 & $36.42-37.33$ & 74.84 & $74.06-75.62$ & $<0.001$ \\
\hline Age (years) & 41.7 & $41.0-42.4$ & 50.9 & $50.0-51.7$ & 48.0 & $46.7-49.3$ & $<0.001$ \\
\hline Sex, n (\%) & & & & & & & $<0.001$ \\
\hline Male & $2,086(30.2 \%)$ & & $\mathrm{I}, 164(52.2 \%)$ & & $719(62.1 \%)$ & & \\
\hline Female & 4,831 (69.8\%) & & $\mathrm{I}, 065(47.8 \%)$ & & $438(37.9 \%)$ & & \\
\hline Smoking status & & & & & & & $<0.001$ \\
\hline Non-smoker & $4,757(66.6 \%)$ & & $\mathrm{I}, 155(47.0)$ & & 47I (36.6) & & \\
\hline Ex-smoker & I,09I (I5.9\%) & & $571(25.8)$ & & $336(27.5)$ & & \\
\hline Current smoker & 934 (I7.5\%) & & $447(27.2)$ & & $325(35.8)$ & & \\
\hline Body mass index $\left(\mathrm{kg} / \mathrm{m}^{2}\right)$ & 21.5 & $21.4-21.6$ & 25.2 & $25.1-25.3$ & 28.4 & $28.2-28.7$ & $<0.001$ \\
\hline Waist circumference $(\mathrm{cm})$ & 74.0 & $73.7-74.3$ & 86.2 & $85.9-86.5$ & 95.4 & $94.8-96.0$ & $<0.001$ \\
\hline $\mathrm{SBP}(\mathrm{mmHg})$ & 112.8 & $112.2-113.5$ & 123.3 & $122.6-124.1$ & 126.2 & $125.1-127.4$ & $<0.001$ \\
\hline $\mathrm{DBP}(\mathrm{mmHg})$ & 72.9 & $72.5-73.3$ & 79.0 & $78.5-79.5$ & 82.5 & $81.7-83.2$ & $<0.001$ \\
\hline HbAlc (\%) & 5.55 & $5.51-5.59$ & 6.00 & $5.93-6.08$ & 6.30 & $6.18-6.42$ & $<0.001$ \\
\hline Fasting glucose $(\mathrm{mg} / \mathrm{dL})$ & 90.7 & $90.2-91.2$ & 100.3 & $99.2-101.3$ & 108.5 & $106.4-110.6$ & $<0.001$ \\
\hline Insulin $(\mu \mathrm{IU} / \mathrm{mL})$ & 9.3 & $9.0-9.6$ & 11.4 & $11.0-11.8$ & 15.1 & $14.0-16.2$ & $<0.001$ \\
\hline HOMA-IR & 2.12 & $2.03-2.20$ & 2.85 & $2.74-2.96$ & 4.11 & $3.77-4.44$ & $<0.001$ \\
\hline Total cholesterol (mg/dL) & 178.2 & $177.0-179.4$ & 196.6 & $195.1-198.1$ & 205.7 & $203.1-208.4$ & $<0.001$ \\
\hline Triglyceride (mg/dL) & 82.1 & $80.9-83.3$ & 155.2 & $151.6-158.7$ & 249.7 & $236.3-263.0$ & $<0.001$ \\
\hline HDL-cholesterol (mg/dL) & 51.9 & $51.6-52.3$ & 44.6 & $44.2-45.1$ & 41.7 & $41.0-42.4$ & $<0.001$ \\
\hline LDL-cholesterol (mg/dL) & 109.0 & $108.0-110.0$ & 122.5 & $121.1-123.9$ & 121.4 & $118.7-124.0$ & $<0.001$ \\
\hline AST (IU/L) & 19.1 & $18.8-19.4$ & 22.5 & $22.1-22.9$ & 29.1 & $28.0-30.2$ & $<0.001$ \\
\hline ALT (IU/L) & 15.5 & $15.2-15.9$ & 24.0 & $23.3-24.7$ & 37.8 & $35.9-39.7$ & $<0.001$ \\
\hline GGT (IU/L) & 18.0 & $17.6-18.4$ & 33.7 & $32.4-35.1$ & 70.9 & $65.6-76.3$ & $<0.001$ \\
\hline
\end{tabular}

Abbreviations: FLI, fatty liver index; GGT, gamma-glutamyl transferase; HbAlc, hemoglobin Alc; HDL, high-density lipoprotein; HOMA-IR, homeostasis model assessment of insulin resistance; LDL, low-density lipoprotein. 
Table 3 Prevalence rates of hypertension, DM, metabolic syndrome, and cardiovascular disease categorized by FLI

\begin{tabular}{|l|l|l|l|l|}
\hline & FLI $<\mathbf{2 0}(\mathbf{n = 5 , 5 6 5 )}$ & FLI 20-59 (n=3,408) & FLI $\geq \mathbf{6 0}(\mathbf{n = I}, \mathbf{I 3 4 )}$ & $\mathbf{P}$ for trend \\
\hline Hypertension & $\mathrm{I}, 059(14.4)$ & $\mathrm{I}, 536(38.0)$ & $628(49.7)$ & $<0.00 \mathrm{I}$ \\
\hline DM, $\mathrm{n}(\%)$ & $265(3.8)$ & $498(I 1.7)$ & $273(20.4)$ & $<0.00 \mathrm{I}$ \\
\hline Metabolic syndrome, $\mathrm{n}(\%)$ & $547(7.4)$ & $\mathrm{I}, 740(43.6)$ & $892(74.9)$ & $<0.00 \mathrm{I}$ \\
\hline Cardiovascular disease, $\mathrm{n}(\%)$ & $\mathrm{I} 6 \mathrm{I}(2.0)$ & $268(5.8)$ & $77(4.7)$ & $<0.00 \mathrm{I}$ \\
\hline
\end{tabular}

Abbreviations: DM, diabetes mellitus; FLI, fatty liver index.

Table 4 The association of hypertension, diabetes mellitus, metabolic syndrome, and cardiovascular disease with FLI

\begin{tabular}{|c|c|c|c|c|c|c|}
\hline & $\begin{array}{l}\text { FLI }<20 \\
(n=5,565)\end{array}$ & $\begin{array}{l}\text { FLI } 20-59 \\
(n=3,408)\end{array}$ & $95 \% \mathrm{Cl}$ & $\begin{array}{l}\text { FLI } \geq 60 \\
(n=I, I 34)\end{array}$ & $95 \% \mathrm{Cl}$ & Nagelkerke $R^{2}$ \\
\hline \multicolumn{7}{|c|}{ Hypertension } \\
\hline Model I & 1.00 & 2.49 & $2.19-2.84$ & 5.57 & $4.56-6.80$ & 0.365 \\
\hline Model 2 & 1.00 & 2.62 & $2.29-2.99$ & 6.27 & $5.05-7.77$ & 0.367 \\
\hline Model 3 & 1.00 & 1.76 & $1.48-2.10$ & 2.92 & $2.18-3.90$ & 0.380 \\
\hline \multicolumn{7}{|c|}{ Diabetes mellitus } \\
\hline Model I & 1.00 & 2.27 & $1.83-2.81$ & 5.49 & $4.19-7.19$ & 0.202 \\
\hline Model 2 & 1.00 & 2.26 & I.82-2.82 & 5.25 & $3.96-6.96$ & 0.211 \\
\hline Model 3 & 1.00 & 2.09 & $1.60-2.71$ & 4.38 & $2.96-6.49$ & 0.218 \\
\hline \multicolumn{7}{|c|}{ Metabolic syndrome } \\
\hline Model I & 1.00 & 10.90 & $9.36-12.69$ & 83.25 & 63.39-109.33 & 0.528 \\
\hline Model 2 & 1.00 & 8.82 & 7.47-10.4I & 62.20 & $47.09-82.19$ & 0.569 \\
\hline Model 3 & 1.00 & 5.52 & $4.39-6.95$ & 24.85 & $17.33-35.64$ & 0.643 \\
\hline \multicolumn{7}{|c|}{ Cardiovascular disease } \\
\hline Model I & 1.00 & 1.9 & $1.45-2.50$ & 1.90 & $1.32-2.64$ & 0.183 \\
\hline Model 2 & 1.00 & 1.85 & 1.39-2.47 & 1.42 & $0.96-2.12$ & 0.202 \\
\hline Model 3 & 1.00 & 1.28 & $0.91-1.82$ & 0.72 & $0.40-1.29$ & 0.211 \\
\hline
\end{tabular}

Notes: Model I: adjusted for age and sex. Model 2: adjusted for age, sex, HDL-cholesterol, and LDL-cholesterol. Model 3: adjusted for age, sex, HDL-cholesterol, LDLcholesterol, fasting glucose, body mass index, smoking status with hypertension. Adjusted for age, sex, HDL-cholesterol, LDL-cholesterol, SBP and DBP, body mass index, smoking status with diabetes mellitus. Adjusted for age, sex, HDL-cholesterol, LDL-cholesterol, fasting glucose, SBP and DBP, body mass index, smoking status with metabolic syndrome and cardiovascular disease.

Abbreviations: FLI, fatty liver index; LDL, low-density lipoprotein; HDL, high-density lipoprotein.

(95\% CI $=4.19-7.19)$ for $\mathrm{DM}, 83.25(95 \% \mathrm{CI}=63.39$ 109.33) for MetS, and 1.90 (95\% CI =1.32-0.64) for CVD. Multiple logistic regression analysis performed by adjusting age, sex, HDL-cholesterol, and LDL-C revealed that, the patients with higher FLI scores showed a significantly higher prevalence of hypertension and DM (in model 2, in the third category of the FLI: OR $=6.27,95 \% \mathrm{CI}=5.05-7.77$ and $\mathrm{OR}=5.25,95 \% \mathrm{CI}=3.96-6.96$ for hypertension and $\mathrm{DM}$, respectively). In line with this, patients with higher FLI scores also showed higher prevalence of the MetS after adjustment for age, sex, and BMI (in the third category of the FLI: OR $=62.20 ; 95 \%$ CI $=47.09-82.19$ ). The ORs for hypertension, DM, and MetS significantly increased in the higher FLI categories after further adjustments of fasting glucose, SBP and DPBP, BMI, and smoking status (model 3). However, the OR for CVD did not increase significantly according to the FLI category after further adjustments of other risk factors (models 2 and 3 ).

The model for FLI presented an AUC value of $0.849(95 \%$ $\mathrm{CI}=0.841-0.856$ ) for the prediction of MetS. It presented the best AUC value than any other component of the MetS (Table 5). This result implicated that a fatty liver is definitely hepatic and a much important manifestation of MetS. In addition, an FLI score $<20$ showed a good sensitivity ( 0.828$)$ and proved to have a negative-predictive value of $91.9 \%$ for the MetS.

\section{Discussion}

We evaluated the associations between the FLI and hypertension, DM, MetS, and CVD in a large Korean population 
Table 5 (A) The AUC for the prediction of metabolic syndrome by the FLI and each component of metabolic syndrome; (B, C) selecting the optimal cutoff value of FLI in identifying metabolic syndrome

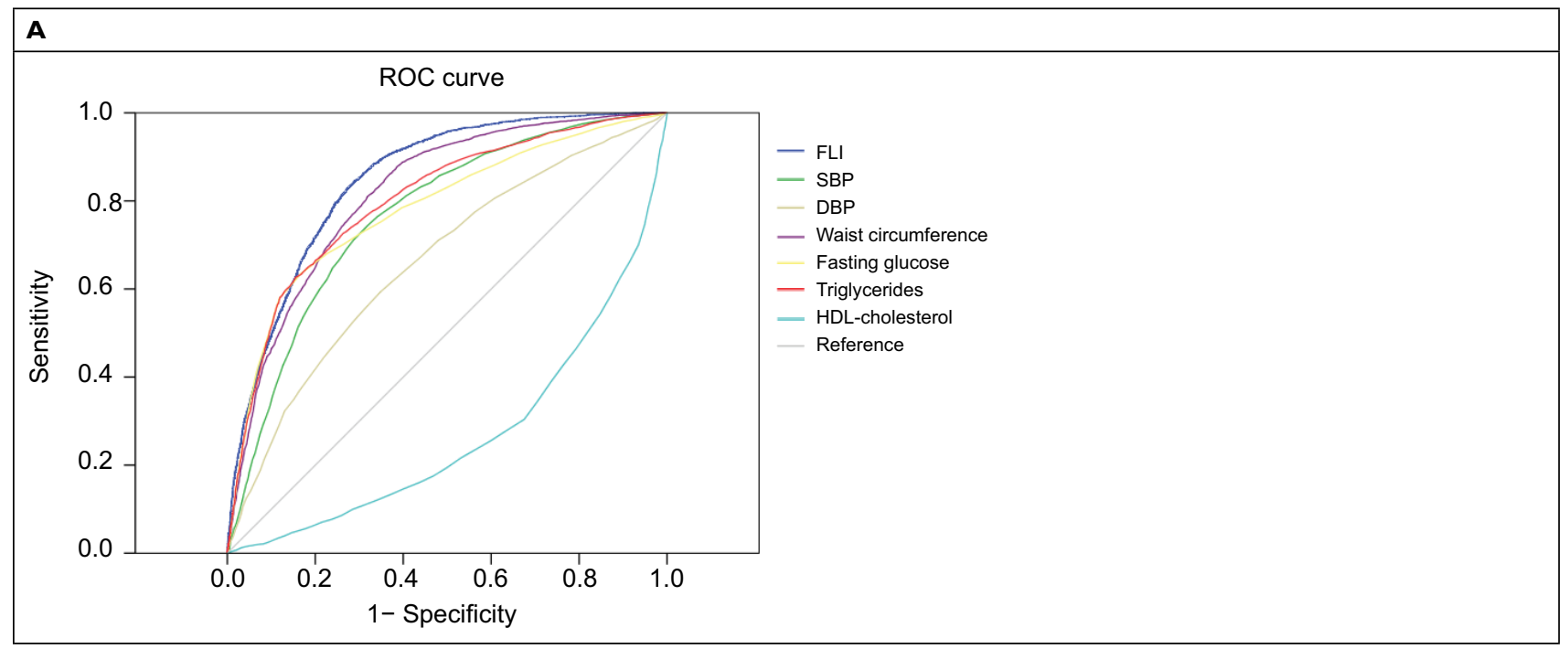

\begin{tabular}{|l|l|l|l|}
\hline B & AUC & $\mathbf{9 5 \%} \mathbf{~ C l}$ & P-value \\
\hline Variable & 0.849 & $0.841-0.857$ & $<0.001$ \\
\hline FLI & 0.769 & $0.759-0.778$ & $<0.001$ \\
\hline SBP & 0.663 & $0.651-0.674$ & $<0.001$ \\
\hline DBP & 0.818 & $0.809-0.826$ & $<0.001$ \\
\hline Waist circumference & 0.781 & $0.771-0.791$ & $<0.001$ \\
\hline Fasting glucose & 0.802 & $0.792-0.811$ & $<0.001$ \\
\hline Triglyceride & 0.270 & $0.259-0.281$ & $<0.001$ \\
\hline HDL-cholesterol & & \\
\hline
\end{tabular}

\begin{tabular}{|l|l|l|l|l|l|l|l|}
\hline C & AUC (95\% CI) & Sensitivity & I - Specificity & $\begin{array}{l}\text { Positive } \\
\text { likelihood } \\
\text { ratio }\end{array}$ & $\begin{array}{l}\text { Negative } \\
\text { likelihood } \\
\text { ratio }\end{array}$ & PPV (\%) & NPV (\%) \\
\hline FLI & $0.849(0.841-0.856)$ & & & & & & \\
\hline 20 & & 0.828 & 0.276 & 3.00 & 0.24 & 52.6 & 91.9 \\
\hline 30 & & 0.668 & 0.171 & 3.91 & 0.40 & 59.1 & 87.1 \\
\hline 60 & & 0.280 & 0.035 & 8.00 & 0.75 & 74.7 & 78.4 \\
\hline
\end{tabular}

Abbreviations: AUC, area under the receiver operating characteristic curve; FLI, fatty liver index; HDL, high-density lipoprotein; NPV, negative predictive value; PPV, positive predictive value; ROC, receiver operating characteristic.

$(\mathrm{N}=10,107)$. Higher FLI, used as an estimate of the presence of fatty liver, showed higher prevalence of hypertension, DM, and MetS after adjustment of other metabolic risk factors. The association between the FLI and MetS was independent from each of the components of the MetS. This suggests that a fatty liver is an important manifestation of MetS independent of dyslipidemia, insulin resistance, and high blood pressure. The cutoff value of the FLI estimated to predict the presence of MetS is 20. Although a higher FLI has been apparently associated with a higher prevalence of metabolic disturbances, it has not been observed to be associated with CVD after adjustment of other risk factors.

FLI, calculated by using four variables which can be obtained easily, may reduce the medical and economic burden of screening or diagnosing NAFLD, and furthermore, of NAFLD-related morbidity and mortality. The FLI calculation and follow-up of the changes over time may help to decide between the liver US and biopsy to confirm the diagnosis of NAFLD and to estimate the risk for type 2 diabetes, dyslipidemia, and MetS. The diagnostic tools such as liver 
MRS and US are quite expensive; and the liver biopsy for the diagnostic confirmation is an invasive procedure, which has a possibility of complications. There are several preexisting predictive equations for NAFLD, for example, FLI, lipid accumulation products (LAP), NAFLD liver fat score, and liver fat (\%). ${ }^{9,13,14}$ The equation of NAFLD liver fat score and liver fat $(\%)$ require previous history of diabetes and MetS for calculating the equations. FLI and LAP are good predictive tools for NAFLD and are based on simple clinical and biochemical parameters. We think that FLI is an appropriate predictive equation for epidemiologic study or an office setting compared with the other scores, as it has been previously studied worldwide and has been validated in Asia. ${ }^{10,11,15}$ FLI had the best discriminative ability compared to LAP to predict ultrasonographic fatty liver in Taiwanese subjects. ${ }^{10}$ In the Taiwanese and Chinese populations, FLI could detect NAFLD accurately with a good AUC, but the optimal cutoff point of FLI to diagnose NAFLD was lower than that in Western countries. ${ }^{10,11}$ A high FLI score with other metabolic disturbances such as obesity, insulin resistance, and dyslipidemia favors higher potential of nonalcoholic steatohepatitis (NASH) because the progression from NAFLD to NASH accelerates the metabolic disturbances. The patients, especially without any abnormal presenting symptoms, may consider their metabolic disturbances more seriously and check the progression of metabolic abnormality more vigilantly following the score system of the FLI. Therefore, FLI is an appropriate tool to screen for the hepatic manifestations of the MetS that need earlier education, intervention, and medication.

The average global prevalence of NAFLD is $25.3 \%$, with the highest prevalence in the Middle east and South America and the lowest prevalence in Africa. ${ }^{16}$ In Asia, the prevalence of NAFLD was reported to be $27.4 \%$ in the meta-analysis of some studies that were conducted using imaging to diagnose NAFLD. ${ }^{16}$ It included six Korean studies to show the prevalence of NAFLD, which presented the results varying from $23.7 \%$ to $40.2 \% .{ }^{16}$ This variability was compounded by the technology and methodology used to establish the diagnosis of NAFLD. In this study, the ageadjusted prevalence of NAFLD was only $10.0 \%$ estimated by the FLI $\geq 60$, showing much lower rate than the average global prevalence of NAFLD. Although there were different clinical features of fatty liver disease according to ethnicity and the different cutoff values of WC and BMI for Asian populations, FLI previously showed good AUC values for predicting NAFLD. ${ }^{10,11,15}$ FLI needs to be further validated in Korean adults, but an important cause for the lower rate compared to the prevalence of NAFLD in the meta-analysis was the cutoff value rather than specific parameters.

MetS is a cluster of metabolic abnormalities that is used to identify people at risk of CVD. Patients with MetS frequently manifest an excessive fat accumulation in the liver. NAFLD and MetS overlap in many respects, depicting a spectrum of diseases, such as insulin resistance, dyslipidemia, and CVD. The prevalence of both MetS and NAFLD increases with obesity. The presence of both the disorders can predict type 2 diabetes, CVD, NASH, and hepatocellular carcinoma. The liver is, therefore, a key determinant of the metabolic abnormalities. We tried to estimate the FLI cutoff score for MetS, as the FLI-diagnosed NAFLD patients had worse metabolic disturbances than the US-diagnosed NAFLD patients. ${ }^{11}$ Consequently, a value $<20$ was estimated as the cutoff score of the FLI to rule out the MetS, which can be used to screen fatty liver and to select subjects for further evaluation of the MetS. This is much lower than 30, which has been suggested as the cutoff score of the FLI to rule out NAFLD by Bedogni et al. ${ }^{9}$ Gastaldelli et al also reported that the likelihood of not having fatty liver was determined to be $>91 \%$ in a large European population by using the FLI $<20 .{ }^{8}$ The cutoff score of FLI to define the presence of MetS was suggested by Lerchbaum et al to compare polycystic ovary syndrome patients and BMI-matched control women, which revealed 34.6 and 25.0 as good results of sensitivity and specificity, respectively. ${ }^{17}$ The proper cutoff score of FLI to predict MetS in each ethnicity and nation might be a useful tool to identify subjects at high risk for metabolic disturbances.

This study also reported that the FLI was much higher in the Korean population with DM as compared to that in those without DM. The subjects with a higher FLI of 10 presented a $31 \%$ increased risk for DM after adjustment of the various factors. In the EPIC-Potsdam study, the subjects with FLI $\geq 60$ as compared to those with FLI $<30$ presented a multivariable-adjusted HR for DM of 17.6 in females and 10.9 in males. ${ }^{18}$ The patients suffering from type 2 diabetes manifested an $80 \%$ more liver fat than that manifested by the matched controls in a large case-control study. ${ }^{19}$ Additionally, changes in the liver fat content might modify the risk of type 2 diabetes. The progression of fatty liver during a 5-year follow-up was reported to be associated with an increased risk for type 2 diabetes, independent of other risk factors. ${ }^{20}$ Balkau et $\mathrm{al}^{21}$ in France and Jung et $\mathrm{al}^{22}$ in Republic of Korea used FLI $<20$ as the cutoff score to rule out fatty liver, and the OR for the development of diabetes was significantly higher in the group of subjects with a higher FLI. These results support the fact that fatty liver screening by FLI may 
help in the earlier detection of metabolic disturbances such as DM, and that it is important to determine the cutoff value of the FLI for the screening process.

Gastaldelli et $\mathrm{al}^{8}$ reported that an increased intima media thickness (IMT), an increased coronary heart disease risk, and reduced insulin sensitivity are associated with high values of the FLI in a European Caucasian population. Moreover, NAFLD is associated with greater overall mortality and can independently predict the risk of future CVD events. ${ }^{23}$ Carotid IMT has been reported to be associated to liver disease: the highest value was found in the patients with NAFLD as compared to that in the controls and patients with hepatitis virus. ${ }^{24}$ The associations between liver disease and carotid atherosclerosis are independent of classical risk factors, MetS components, and insulin resistance. ${ }^{8,25,26}$ There are many risk factors for CVDs, including high glucose, high blood pressure, high cholesterol, history of smoking, family history, and so on. Since the FLI equation includes TG, GGT, and WC, which are also important risk factors for CVDs, the FLI can also be used as an additional index for accelerated atherosclerosis and cardiovascular risk. However, this study could not show the definite association between CVD and FLI, since the KNHANES study had surveyed for previous CVD history with only questionnaires and without any other atherosclerosis markers such as IMT or treadmill test.

This study has some limitations. One is the lack of US data for the assessment of hepatic steatosis, due to which we were unable to compare between the FLI categories and the real US data. However, FLI validation for Asians has already been reported in South Korea, which showed a good diagnostic accuracy for fatty liver. ${ }^{10,11,15}$ Data on HbA1c and insulin were incomplete (only available for all adults in 2011 KNHANES and for only diabetic patients in 2010 KNHANES), and therefore, we considered all data with respect to personal history, antidiabetic medication, and fasting plasma glucose to define DM status. Another limitation is that only self-reported information was included regarding the history or status of CVD in KNHANES. Therefore, we need further evaluation of FLI as a predictor of CVD in Asians.

\section{Conclusion}

We tried to estimate NAFLD prevalence using FLI in Korean adults, and it was significantly higher in subjects with MetS, supporting that NAFLD is the hepatic manifestation of MetS. Known cardiovascular risk factors, such as obesity, high blood pressure, increased cholesterol, and decreased
HDL cholesterol, show definite associations with higher FLI. FLI might be a useful screening tool to select subjects for liver US, intensified lifestyle modification, and medication in patients with MetS, who have high cardiovascular risk. Further research is needed to determine whether FLI could be a good screening tool for MetS and a predictor for CVD in a clinical setting. Additionally, further studies are required to show if metabolic improvements can be seen with lowering of the FLI.

\section{Author contributions}

ARK, HWL, DWY, YHK, and SMS made substantial contributions to the conception and design, acquisition of data, and analysis and interpretation of data. All authors contributed to data analysis, drafting or revising the article, gave final approval of the version to be published, and agree to be accountable for all aspects of the work.

\section{Disclosure}

The authors report no conflicts of interest in this work.

\section{References}

1. Browning JD, Szczepaniak LS, Dobbins R, et al. Prevalence of hepatic steatosis in an urban population in the United States: impact of ethnicity. Hepatology. 2004;40(6):1387-1395.

2. Bedogni G, Miglioli L, Masutti F, Tiribelli C, Marchesini G, Bellentani S. Prevalence of and risk factors for nonalcoholic fatty liver disease: the Dionysos Nutrition and Liver Study. Hepatology. 2005;42(1):44-52.

3. Ahmed MH, Abu EO, Byrne CD. Non-alcoholic fatty liver disease (NAFLD): new challenge for general practitioners and important burden for health authorities? Prim Care Diabetes. 2010;4(3):129-137.

4. Ahmed MH, Byrne CD. Current treatment of non-alcoholic fatty liver disease. Diabetes Obes Metab. 2009;11(3):188-195.

5. Targher G, Bertolini L, Rodella S, et al. Nonalcoholic fatty liver disease is independently associated with an increased incidence of cardiovascular events in type 2 diabetic patients. Diabetes Care. 2007;30(8): 2119-2121.

6. Ahmed MH. Biochemical markers: the road map for the diagnosis of nonalcoholic fatty liver disease. Am J Clin Pathol. 2007;127(1):20-22.

7. Otgonsuren M, Estep MJ, Hossain N, et al. Single non-invasive model to diagnose non-alcoholic fatty liver disease (NAFLD) and non-alcoholic steatohepatitis (NASH). J Gastroenterol Hepatol. 2014;29(12):2006-2013.

8. Gastaldelli A, Kozakova M, Højlund K, et al; RISC Investigators. Fatty liver is associated with insulin resistance, risk of coronary heart disease, and early atherosclerosis in a large European population. Hepatology. 2009;49(5):1537-1544.

9. Bedogni G, Bellentani S, Miglioli L, et al. The fatty liver index: a simple and accurate predictor of hepatic steatosis in the general population. BMC Gastroenterol. 2006;6(1):33.

10. Yang BL, Wu WC, Fang KC, et al. External validation of fatty liver index for identifying ultrasonographic fatty liver in a large-scale crosssectional study in Taiwan. PLoS One. 2015;10(3):e0120443.

11. Huang $\mathrm{X}, \mathrm{Xu} \mathrm{M}, \mathrm{Chen} \mathrm{Y}$, et al. Validation of the fatty liver index for nonalcoholic fatty liver disease in middle-aged and elderly Chinese. Medicine (Baltimore). 2015;94(40):e1682.

12. Ford ES, Giles WH, Mokdad AH. Increasing prevalence of the metabolic syndrome among U.S. adults. Diabetes Care. 2004;27(10):2444-2449. 
13. Bedogni G, Kahn HS, Bellentani S, Tiribelli C. A simple index of lipid overaccumulation is a good marker of liver steatosis. BMC Gastroenterol. 2010;10(1):98.

14. Kotronen A, Peltonen M, Hakkarainen A, et al. Prediction of nonalcoholic fatty liver disease and liver fat using metabolic and genetic factors. Gastroenterology. 2009;137(3):865-872.

15. Kim JH, Kwon SY, Lee SW, Lee CH. Validation of fatty liver index and lipid accumulation product for predicting fatty liver in Korean population. Liver Int. 2011;31(10):1600-1601.

16. Younossi ZM, Koenig AB, Abdelatif D, Fazel Y, Henry L, Wymer M. Global epidemiology of nonalcoholic fatty liver disease - meta-analytic assessment of prevalence, incidence, and outcomes. Hepatology. 2016;64(1):73-84.

17. Lerchbaum E, Gruber HJ, Schwetz V, et al. Fatty liver index in polycystic ovary syndrome. Eur J Endocrinol. 2011;165(6):935-943.

18. Jäger S, Jacobs S, Kröger J, et al. Association between the fatty liver index and risk of type 2 diabetes in the EPIC-Potsdam study. PLoS One. 2015;10(4):e0124749.

19. Kotronen A, Juurinen L, Hakkarainen A, et al. Liver fat is increased in type 2 diabetic patients and underestimated by serum alanine aminotransferase compared with equally obese nondiabetic subjects. Diabetes Care. 2008;31(1):165-169.
20. Sung KC, Wild SH, Byrne CD. Resolution of fatty liver and risk of incident diabetes. J Clin Endocrinol Metab. 2013;98(9):3637-3643.

21. Balkau B, Lange C, Vol S, Fumeron F, Bonnet F; Group Study D.E.S.I.R. Nine-year incident diabetes is predicted by fatty liver indices: the French D.E.S.I.R. study. BMC Gastroenterol. 2010;10(1):56.

22. Jung CH, Lee WJ, Hwang JY, et al. Assessment of the fatty liver index as an indicator of hepatic steatosis for predicting incident diabetes independently of insulin resistance in a Korean population. Diabet Med. 2013;30(4):428-435.

23. Adams LA, Lymp JF, St Sauver J, et al. The natural history of nonalcoholic fatty liver disease: a population-based cohort study. Gastroenterology. 2005;129(1):113-121.

24. Targher G, Arcaro G. Non-alcoholic fatty liver disease and increased risk of cardiovascular disease. Atherosclerosis. 2007;191(2): 235-240.

25. Brea A, Mosquera D, Martín E, Arizti A, Cordero JL, Ros E. Nonalcoholic fatty liver disease is associated with carotid atherosclerosis: a casecontrol study. Arterioscler Thromb Vasc Biol. 2005;25(5):1045-1050.

26. Völzke H, Robinson DM, Kleine V, et al. Hepatic steatosis is associated with an increased risk of carotid atherosclerosis. World J Gastroenterol. 2005;11(12):1848.
Diabetes, Metabolic Syndrome and Obesity: Targets and Therapy is an international, peer-reviewed open-access journal committed to the rapid publication of the latest laboratory and clinical findings in the fields of diabetes, metabolic syndrome and obesity research. Original research, review, case reports, hypothesis formation, expert opinion and commentaries are all considered for publication. The manuscript management system is completely online and includes a very quick and fair peer-review system, which is all easy to use. Visit http://www.dovepress.com/testimonials.php to read real quotes from published authors. 